Accepted for publication in Ap\&SS

\title{
Investigating thermal evolution of the self-gravitating one dimensional molecular cloud by smoothed particle hydrodynamics
}

\author{
Mohsen Nejad-Asghar ${ }^{1,2}$ and Diego Molteni ${ }^{3}$ \\ ${ }^{1}$ Department of Physics, Damghan University of Basic Sciences, Damghan, Iran \\ ${ }^{2}$ Research Institute for Astronomy and Astrophysics of Maragha, Maragha, Iran \\ ${ }^{3}$ Dipartimento di Fisica e Tecnologie Relative, Universita di Palermo, Viale delle Scienze, \\ 90128, Palermo, Italy
}

\begin{abstract}
The heating of the ion-neutral (or ambipolar) diffusion may affect the thermal phases of the molecular clouds. We present an investigation on the effect of this heating mechanism in the thermal instability of the molecular clouds. A weakly ionized one dimensional slab geometry, which is allowed for self-gravity and ambipolar diffusion, is chosen to study its thermal phases. We use the thermodynamic evolution of the slab to obtain the regions where slab cloud becomes thermally unstable. We investigate this evolution using the model of ambipolar diffusion with two-fluid smoothed particle hydrodynamics, as outlined by Hosking \& Whitworth. Firstly, some parts of the technique are improved to test the pioneer works on behavior of the ambipolar diffusion in an isothermal self-gravitating slab. Afterwards, the improved two-fluid technique is used for thermal evolution of the slab. The results show that the thermal instability may persist inhomogeneities with a large density contrast at the intermediate parts of the cloud. We suggest that this feature may be responsible for the planet formation in the intermediate regions of a collapsing molecular cloud and/or may also be relevant to the formation of star forming dense cores in the clumps.
\end{abstract}

Subject headings: ISM: clouds - Hydrodynamics - ISM: magnetic fields - diffusion - methods: numerical - ISM: evolution. 


\section{Introduction}

Observations of molecular clouds show the existence of small density fluctuations threaded by magnetic fields. The recent progress of the molecular cloud observations has established that the small and tiny scale structures are very ubiquitous. For example, Langer et al. (1995) observed small condensations with size from 0.007 to $0.021 \mathrm{pc}$ in Taurus Molecular Cloud 1. The mass of these fragments is estimated to be $<0.01-0.15 M_{\odot}$. Studies of the time variability of absorption lines indicates the presence of fluctuations on scales of $10^{-4} \mathrm{pc}(5-50$ AU) and masses of $10^{-9} \mathrm{M}_{\odot}$ (Boissé et al. 2005). At larger scales (about 10,000 AU), Pan et al. (2001) find significant differences in $\mathrm{CN}, \mathrm{CH}$, and $\mathrm{CH}^{+}$absorption lines. The sPITzER experiment has begun producing higher spatial resolution mid-infrared maps (Churchwell et al. 2004) and revealed the fine structure of the star forming regions. The advent of major new facilities in the coming years should yield several breakthroughs in this field (André et al. 2008). A higher resolution observation in future is assuredly expected to reveal hidden, small structures, as well as sub-stellar objects with very small mass (Atacama Large Millimeter Array, ALmA, becoming partly available in 2011, fully operational at 2013).

The classical problems of cloud fragmentation are relevant to star formation because they show that there is a preferred scale (Jeans length) of gravitational instability as soon as one considers an initial structured region with some length-scale (see, e.g., Larson 1985). By the way, it is not easy for the small and tiny scale structure to form as a result of gravitational instability, since their size are much smaller than the Jeans length. According to Langer et al. (1995) indeed, the small scale structures appear to be gravitationally unbound. This suggests that some fragmentation mechanisms other than pure Jeans gravitational instability may be important in the clouds. Molecular clouds are thought to be supersonically turbulent, since they exhibit supersonic linewidths (Zuckerman \& Palmer 1974). Base on the idea of turbulence, some mechanisms to generate substructures inside molecular clouds have been proposed (Elmegreen 2007). As well, a further process based on magnetohydrodynamic waves have been suggested to produce inhomogeneities in the clouds (Folini et al. 2004). Another basic physical process that could trigger formation of density fluctuations is thermal instability, because it acts on time-scales that can be much shorter than the duration of the turbulent motions (Gilden 1984). The effect of thermal instability in fragmentation of the clouds is clearly a competitive process relative to other mechanisms, although all could operate.

In molecular clouds, a neutral molecular gas is intermixed with an ionized component that is tied directly to the magnetic field. Birk (2000) has used the two-fluid technique to find the thermal condensation modes in weakly ionized hydrogen plasma. Nejad-Asghar \& Ghanbari (2003) studied the effect of linear thermal instability in a weakly ionized magnetic 
molecular cloud within the one-fluid description. Nejad-Asghar (2007) has recently made the assumption that the molecular cloud is initially an uniform ensemble which then fragments due to thermal instability. He find that ambipolar drift heating is inversely proportional to density and its value, in outer parts of the cloud, can be significantly larger than the average heating rates of cosmic rays and turbulent motions. His results show that the isobaric thermal instability can occur in intermediate regions of the cloud; therefore it may produce the cloud fragmentation and formation of the condensations.

The study under the one-fluid approach did not precisely consider the effect of the ionneutral friction, since they did not treat the flow as two fluids which are composed of ions and neutrals. Then, Fukue \& Kamaya (2007) revisited the effect of the ion-neutral friction of the two-fluid on the growth of the linear thermal instability. Their results indicate that the friction with the magnetic field affects the morphology and evolution of the interstellar matter. Falle et al. (2006) described numerical calculations using an AMR magnetohydrodynamic code that show that thermal instability may have an important role to play in the formation of the hierarchical structure of molecular clouds. Here, we use the two-fluid numerical simulation to study the evolution of weakly ionized molecular clouds in the nonlinear thermal instability regime.

Many authors have developed computer codes that attempt to model ion-neutral diffusion. Black \& Scott (1982) used a two-dimensional, deformable-grid algorithm to follow the collapse of isothermal, non-rotating magnetized cloud. The three-dimensional work of MacLow et al. (1995) treats the two-fluid model in a version of the ZEUs magnetohydrodynamic code. An algorithm capable of using the smoothed particle hydrodynamics (SPH) to implement the ambipolar diffusion in a fully three-dimensional, self-gravitating system was developed by Hosking \& Whitworth (2004, hereafter HW). They described the SPH implementation of two-fluid technique that was tested by modeling the evolution of a dense core, which is initially thermally supercritical but magnetically subcritical.

In this paper, we firstly re-formulate and improve the two-fluid SPH implementation of the ambipolar diffusion in an isothermal self-gravitating one dimensional slab. Afterwards, we use this two-fluid SPH technique to investigate the nonlinear thermal evolution of the slab. In this way, the continuum equations of the self-gravitating slab and its isothermal case are given is section 2. The two-fluid SPH technique is presented in section 3. Section 4 devoted to the chosen physical scales, initial conditions, and the computer experiments to explore the temperature profile and density fluctuations in the contracting one dimensional molecular cloud. Finally, section 5 is allocated to the summary and conclusions with some prospects. 


\section{Gas dynamics}

In the absence of reliable information about the structure of the molecular clouds, it is reasonable to examine simple geometries such as sphere, disks, cylinder, etc., in order to gain some insight into the evolution process. Here, we consider an one-dimensional lightly ionized molecular gas with purely transverse magnetic field, thus, all variables are functions of distance $z$ to the center and time $t$ only.

\subsection{The continuum equations}

The exact fraction of the total fluid that is ionized depends upon many factors (e.g. the neutral density, the cosmic ray ionization rate, how efficiently ionized metals are depleted on to dust grains). Here, we use the expression employed by Fiedler \& Mouschovias (1992), which states that for $10^{8}<n<10^{15} \mathrm{~m}^{-3}$,

$$
\rho_{i}=\epsilon\left(\rho^{1 / 2}+\epsilon^{\prime} \rho^{-2}\right)
$$

where in standard ionized equilibrium state, $\epsilon \sim 7.5 \times 10^{-15} \mathrm{~kg}^{1 / 2} \cdot \mathrm{m}^{-3 / 2}$ and $\epsilon^{\prime} \sim 4 \times$ $10^{-44} \mathrm{~kg}^{5 / 2} \cdot \mathrm{m}^{-15 / 2}$ are valid. In reality, the gas in this case is very weakly ionized, thus, we adopt the approximation $\rho=\rho_{n}+\rho_{i} \approx \rho_{n}$ in fluid equations. In this case, the molecular cloud is considered as global neutral which consists of a mixture of atomic and molecular hydrogen (with mass fraction $X$ ), helium (with mass fraction $Y$ ), and traces of CO and other rare molecules, thus, the mean molecular weight is given by $1 / \mu=X / 2+Y / 4$.

We write the continuity equation of neutral part as its common form

$$
\frac{d \rho}{d t}=-\rho \frac{\partial v}{\partial z}
$$

while, the relation (11) is used to determine the ion density wherever it is required in the two-fluid equations. The momentum equation of neutral part then becomes

$$
\frac{d v}{d t}=g-\frac{1}{\rho} \frac{\partial}{\partial z}\left(p+\frac{B^{2}}{2 \mu_{0}}\right)
$$

where the gravitational acceleration $g$ obeys the poisson's equation

$$
\frac{\partial g}{\partial z}=-4 \pi G \rho
$$

and the pressure is given by the ideal gas equation of state

$$
p=\frac{R}{\mu} \rho T
$$


where $R$ is the molar gas constant and the temperature $T$ is approximated the same as for both neutral and ion fluids $\left(T_{i}=T_{n}=T\right)$.

The calculations are usually further simplified by the assumption of an isothermal equation of state. This last assumption is, however, unnecessarily crude since molecular gas is expected to cool and heat rapidly and appropriate cooling and heating function may be estimated (e.g., Goldsmith 2001). The thermal energy per unit mass is generally given by

$$
u(T)=\left(\frac{5}{4} X+\frac{3}{8} Y\right) \frac{k_{B} T}{m_{H}}
$$

where the mean internal (rotation and vibration) energy of an $\mathrm{H}_{2}$ molecule is included. At temperature below $\sim 200 \mathrm{~K}$, the rotational and vibration degrees of freedom of molecular hydrogen are not excited, so it actually behaves like a monatomic gas. The energy equation follows from the first law of thermodynamics, that is

$$
\frac{d u}{d t}=-\frac{p}{\rho} \frac{\partial v}{\partial z}-\Omega_{(\rho, T)}
$$

where $\Omega_{(\rho, T)}$ is the net cooling function

$$
\Omega_{(\rho, T)} \equiv \Lambda_{(n)}\left(\frac{T}{10 \mathrm{~K}}\right)^{\beta_{(n)}}-\left(\Gamma_{C R}+\Gamma_{A D}\right),
$$

where $\Gamma_{C R}$ and $\Gamma_{A D}$ are the heating rates due to cosmic rays and ambipolar diffusion, respectively, and $\Lambda_{(n)}$ and $\beta_{(n)}$ are the parameters for the gas cooling function that here we use the polynomial fitting functions, outlined by Nejad-Asghar (2007), as follows

$$
\begin{gathered}
\log \left(\frac{\Lambda_{(n)}}{{\mathrm{J} . \mathrm{kg}^{-1} \cdot \mathrm{S}^{-1}}}\right)=-8.98-0.87\left(\log \frac{n}{n_{0}}\right) \\
-0.14\left(\log \frac{n}{n_{0}}\right)^{2}, \\
\beta_{(n)}=3.07-0.11\left(\log \frac{n}{n_{0}}\right)-0.13\left(\log \frac{n}{n_{0}}\right)^{2},
\end{gathered}
$$

where $n_{0}=10^{12} \mathrm{~m}^{-3}$. The heating of cosmic rays is given by (e.g., Goldsmith 2001),

$$
\Gamma_{C R} \approx 3.12 \times 10^{-8} \quad \mathrm{~J} \cdot \mathrm{kg}^{-1} \cdot \mathrm{s}^{-1},
$$

while the heating due to ambipolar diffusion can be physical derived by considering the drag force (per unit volume) as follows

$$
\Gamma_{A D}=\frac{\mathbf{f}_{d} \cdot \mathbf{v}_{d}}{\rho}
$$

where

$$
\mathbf{f}_{d}=\gamma_{A D} \rho_{i} \rho \mathbf{v}_{d}
$$


where $\gamma_{A D} \sim 3.5 \times 10^{10} \mathrm{~m}^{3} . \mathrm{kg}^{-1} . \mathrm{s}^{-1}$ represents the collision drag coefficient.

The magnetic fields are directly evolved by charged fluid component, as follows:

$$
\frac{d B}{d t}=-B \frac{\partial v}{\partial z}+\frac{\partial}{\partial z}\left(B v_{d}\right)
$$

where the last term outlines the ambipolar diffusion effect with drift velocity,

$$
v_{d}=-\frac{1}{\gamma_{A D} \epsilon \rho \rho_{i}} \frac{\partial}{\partial z}\left(\frac{B^{2}}{2 \mu_{0}}\right),
$$

which is obtained by assumption that the pressure and gravitational force on the charged fluid component are negligible compared to the Lorentz force because of the low ionization fraction.

\subsection{Isothermal molecular layer}

In isothermal case, the thermal energy per unit mass (66) is overplus, and the pressure in momentum equation (3) then becomes $p=a^{2} \rho$ where $a$ is the isothermal sound speed. Following the many previous treatments, a further simplification is possible if we introduce the surface density between mid-plane and $z>0$ as

$$
\sigma \equiv \int_{0}^{z} \rho\left(z^{\prime}, t\right) d z^{\prime}
$$

By transformation from $(z, t)$ to $(\sigma, t)$, the drift velocity is given by

$$
v_{d}=-\frac{1}{\gamma_{A D} \epsilon \rho^{1 / 2}\left(1+\epsilon^{\prime} \rho^{-5 / 2}\right)} \frac{\partial}{\partial \sigma}\left(\frac{B^{2}}{2 \mu_{0}}\right),
$$

and the equation (14) becomes

$$
\frac{\partial}{\partial t}\left(\frac{B}{\rho}\right)=\frac{1}{\gamma_{A D} \epsilon \mu_{0}} \frac{\partial}{\partial \sigma}\left(\frac{B^{2}}{\rho^{1 / 2}\left(1+\epsilon^{\prime} \rho^{-5 / 2}\right)} \frac{\partial B}{\partial \sigma}\right) .
$$

With the above, field equation (4) can be integrated to give

$$
g=-4 \pi G \sigma
$$

while the equation of continuity (2) and the equation of motion (3) take the form

$$
\frac{\partial z}{\partial \sigma}=\frac{1}{\rho}
$$


and

$$
\frac{\partial^{2} z}{\partial t^{2}}=-4 \pi G \sigma-\frac{\partial}{\partial \sigma}\left(a^{2} \rho+\frac{B^{2}}{2 \mu_{0}}\right),
$$

respectively. The slab is assumed to be in quasi-magnetohydrostatic equilibrium at all times, supported against its own self-gravity by the magnetic and gas pressures. The loss of flux from ambipolar diffusion is exactly compensated for by the compression of the slab which is necessary to maintain equilibrium. In this approximation, the left-hand side of equation (21) is zero, and we may integrate the force balance to obtain

$$
\frac{B^{2}}{2 \mu_{0}}+a^{2} \rho=2 \pi G\left(\sigma_{\infty}^{2}-\sigma^{2}\right)
$$

where integration constant $\sigma_{\infty}$ is the value of $\sigma$ at $z=\infty$ (where $\rho$ is zero).

Following the work of Shu (1983), we introduce the non-dimension quantities

$$
\begin{gathered}
\tilde{\sigma} \equiv \frac{\sigma}{\sigma_{\infty}}, \quad \tilde{\rho} \equiv \frac{a^{2}}{2 \pi G \sigma_{\infty}^{2}} \rho, \quad \tilde{z} \equiv \frac{2 \pi G \sigma_{\infty}}{a^{2}} z, \quad \tilde{B} \equiv \frac{B}{2 \sigma_{\infty} \sqrt{\pi \mu_{0} G}} \\
\tilde{t} \equiv\left(\frac{2 \sqrt{2 \pi G}}{\gamma_{A D} \epsilon}\right)\left(\frac{2 \pi G \sigma_{\infty}}{a}\right) t, \quad \tilde{\epsilon} \equiv \frac{a^{5}}{(2 \pi G)^{5 / 2} \sigma_{\infty}^{5}} \epsilon^{\prime}, \quad \tilde{v}_{d} \equiv \frac{\gamma_{A D} \epsilon}{a \sqrt{2 \pi G}} v_{d},
\end{gathered}
$$

so that we rewrite the basic equations (18), (20) and (22) as follows:

$$
\begin{gathered}
\frac{\partial}{\partial \tilde{t}}\left(\frac{\tilde{B}}{\tilde{\rho}}\right)=\frac{\partial}{\partial \tilde{\sigma}}\left(\frac{\tilde{B}^{2}}{\tilde{\rho}^{1 / 2}+\tilde{\epsilon} \tilde{\rho}^{-2}} \frac{\partial \tilde{B}}{\partial \tilde{\sigma}}\right), \\
\tilde{B}^{2}+\tilde{\rho}=1-\tilde{\sigma}^{2} \\
\frac{\partial \tilde{z}}{\partial \tilde{\sigma}}=\frac{1}{\tilde{\rho}}
\end{gathered}
$$

and the drift velocity (17) as

$$
\tilde{v}_{d}=-\frac{1}{\tilde{\rho}^{1 / 2}+\tilde{\epsilon} \tilde{\rho}^{-2}} \frac{\partial \tilde{B}^{2}}{\partial \tilde{\sigma}} .
$$

Here, a natural family of initial states is generated by assuming that the initial ratio of magnetic to gas pressure is everywhere a constant, $\alpha_{0}$, i.e., $\tilde{B}^{2} / \tilde{\rho}=\alpha_{0}$ at $\tilde{t}=0$. Then one finds from equations (25), (26) and (27) that

$$
\begin{gathered}
\rho_{(z, t=0)}=\frac{\rho_{0}}{\cosh ^{2}\left(z / z_{\infty}\right)}, \\
v_{d(z, t=0)}=\frac{2 \alpha_{0}}{\sqrt{1+\alpha_{0}}} \frac{a \sqrt{2 \pi G}}{\gamma_{A D}} \frac{\sinh \left(z / z_{\infty}\right)}{1+\epsilon^{\prime} \rho_{0}^{-5 / 2} \cosh ^{5}\left(z / z_{\infty}\right)},
\end{gathered}
$$


where $\rho_{0} \equiv 2 \pi G \sigma_{\infty}^{2} / a^{2}\left(1+\alpha_{0}\right)$ is the central density of the slab at $t=0$, and $z_{\infty} \equiv$ $a \sqrt{\left(1+\alpha_{0}\right) / 2 \pi G \rho_{0}}$ is a length-scale parameter.

Figure 1 shows the initial neural density, ion density, and drift velocity in the cloud and outercloud medium for $\rho_{0}=3.8 \times 10^{-15} \mathrm{~kg} \cdot \mathrm{m}^{-3}, a=0.55 \mathrm{~km} \cdot \mathrm{s}^{-1}$, and $\alpha_{0}=1$. Shu (1983) solved the equations (24)-(27) by finite difference techniques under the initial and boundary conditions

$$
\tilde{B}=\left(\frac{\alpha_{0}}{1+\alpha_{0}}\right)^{1 / 2}\left(1-\tilde{\sigma}^{2}\right)^{1 / 2} \text { at } \tilde{t}=0 \quad \text { and } \quad \tilde{B}=0 \text { at } \tilde{\sigma}=1 .
$$

The integrations are carried out to time $\tilde{t}=20$ with initial conditions $\alpha_{0}=1$ and 10 , and the results are presented graphically in his paper (Shu 1983). As the magnetic field leaks from the neutral gas, the volume density of the neutrals shifts in profile from equation (28) to that case with $\alpha_{0}=0$, and the drift velocity gradually settles.

\section{Numerical scheme}

Since the molecular gas is expected to cool and heat rapidly, considering the thermal energy is appropriate. For this aim, we produce a two-fluid SPH implementation more efficient than HW, to study the thermal evolution of the self-gravitating one dimensional molecular cloud. In the technique of HW, the initial SPH particles are represented by two sets of particles: magnetized ion SPH particles and non-magnetized neutral SPH particles. For each SPH particle we must create two separate neighbor lists: one for neighbors of the same species and another for those of different species. Consequently, each particle must have two different smoothing lengths. In the following sections we refer to neutral particles as $\alpha$ and $\beta$, and ion particles as $a$ and $b$; the subscripts 1 and 2 refer to both ions and neutral particles.

The neutral density in place of neutral particles is estimated via usual summation over neighboring neutral particles

$$
\rho_{n, \alpha}=\sum_{\beta} m_{\beta} W_{\alpha \beta}
$$

while in place of ions, $\rho_{n, a}$, is given by interpolation technique from the values of nearest neighbors. The ion density is evaluated via equation (1) for both places of ions and neutral particles. In the particle approximation of SPH method, the infinitesimal volume in the integrations at the location of particle $a$ is replaced by the finite volume of that particle $\triangle V_{a}$, which is related to the mass and density as $m_{a}=\rho_{a} \triangle V_{a}$. Since the ion density is 
evaluated by equation (11), no by the usual summation rule

$$
\rho_{i, a}=\sum_{\beta} m_{b} W_{a b}
$$

we must update the mass of ion $a$ as follows

$$
m_{a}^{\text {new }}=m_{a}^{\text {old }} \frac{\rho_{a}^{\text {new }}}{\rho_{a}^{\text {old }}}
$$

in each time step so that the above summation/interpolation routine for ions (32) being accurate.

The SPH form of the drift velocity of ion particle $a$ is given by HW as

$$
v_{d, a}=\frac{1}{\gamma_{A D} \rho_{n, a}}\left[-\frac{1}{\mu_{0} \rho_{i, a}} \sum_{b} \frac{m_{b}}{\rho_{i, b}} B_{b} B_{a} \frac{d W_{a b}}{d z_{a}}-\sum_{b} m_{b} \Pi_{a b} \frac{d W_{a b}}{d z_{a}}\right] .
$$

where $\Pi_{a b}$ is the usual artificial viscosity between ion particles $a$ and $b$ (Monaghan 1992). The artificial viscosity is reconsidered in SPH to prevent inter-particle penetration, unwanted heating, and unphysical solutions. Nejad-Asghar, Khesali \& Soltani (2008) has recently considered the coefficients in the Monaghan's standard artificial viscosity as time variable, and a restriction on them is proposed such that avoiding the undesired effects in the subsonic regions. Here, we use the Monaghan's standard artificial viscosity, since the cloud contraction is quasi-hydrostatic and there is not supersonic motions and shock formation during this contraction. Keeping in mind the second golden rule of SPH which is to rewrite formulae with the density inside operators (Monaghan 1992), we can optimize the drift velocity of HW as follows

$$
\begin{array}{r}
v_{d, a}=\frac{1}{\gamma_{A D} \rho_{n, a}}\left[-\frac{1}{2 \mu_{0} \rho_{i, a}} \sum_{b} \frac{m_{b}}{\rho_{i, b}}\left(B_{b}^{2}-B_{a}^{2}\right) \frac{d W_{a b}}{d z_{a}}\right. \\
\left.-\rho_{i, a} \sum_{b} \frac{m_{b}}{\rho_{i, b}} \prod_{a b} \frac{d W_{a b}}{d z_{a}}\right],
\end{array}
$$

where two extra density terms are introduced, one outside and one inside the summation sign. This comes as a result of the approximation to the volume integral needed to perform function interpolation. The drift velocity at neutral places are used to estimate the drag acceleration

$$
a_{d r a g, \alpha}=\gamma_{A D} \rho_{i, \alpha} v_{d, \alpha}
$$

instead the method of HW who used the expression of Monaghan \& Kocharayan (1995). Since there is no any analytical expression that allows us to calculate the value of drift velocity in place of the neutral particles, we use the interpolation technique that starts at 
the nearest neighbor, then add a sequence of decreasing corrections, as information from other neighbors is incorporated (e.g., Press et al. 1992).

In the usual symmetric form, the self-gravitating SPH acceleration equation for neutral particle $\alpha$ is

$$
\frac{d v_{\alpha}}{d t}=g_{\alpha}-\sum_{\beta} m_{\beta}\left(\frac{p_{\alpha}}{\rho_{\alpha}^{2}}+\frac{p_{\beta}}{\rho_{\beta}^{2}}+\Pi_{\alpha \beta}\right) \frac{d W_{\alpha \beta}}{d z_{\alpha}}+a_{d r a g, \alpha}
$$

where $g_{\alpha}$ is the gravitational acceleration of particle $\alpha$. The ion momentum equation assuming instantaneous velocity update so that we have

$$
v_{a}=\sum_{\beta} \frac{m_{\beta}}{\rho_{\beta}} v_{\beta} W_{a \beta}+v_{d, a}
$$

where the first term on the right-hand side gives the neutral velocity field at the ion particle $a$, calculated using a standard SPH approximation.

The SPH equivalent of the energy equation (7) is

$$
\frac{d u_{\alpha}}{d t}=\frac{1}{2} \sum_{\beta} m_{\beta}\left(\frac{p_{\alpha}}{\rho_{\alpha}^{2}}+\frac{p_{\beta}}{\rho_{\beta}^{2}}+\Pi_{\alpha \beta}\right) v_{\alpha \beta} \frac{\partial W_{\alpha \beta}}{\partial z_{\alpha}}-\Omega_{\alpha} .
$$

The temperatures of neutral particles are calculated from equation (6), and the temperature of any ion is assumed to be same as its nearest neutral neighbor. Finally, the magnetic induction equation (14) in SPH form is replaced by

$$
\frac{d B_{a}}{d t}=\sum_{b} \frac{m_{b}}{\rho_{b}} B_{a} v_{a b} \frac{d W_{a b}}{d z_{a}}
$$

where the usual notations of the ion fluid are used.

\section{The computer experiments}

The chosen physical scales for length and time are $[l]=200 \mathrm{AU}$, and $[t]=10^{3} \mathrm{yr}$, respectively, so that velocity unit is approximately $[v]=1 \mathrm{~km} . \mathrm{s}^{-1}$. The Newtonian constant of gravitation is set $G=1[m]^{-1}[l]^{3}[t]^{-2}$ for which the calculated mass unit is $[m]=4.5 \times$ $10^{29} \mathrm{~kg}$. Consequently, the derived physical scale for density, energy per unit mass, and drag coefficient are $[\rho]=1.7 \times 10^{-11} \mathrm{~kg} \cdot \mathrm{m}^{-3},[u]=10^{6} \mathrm{~J}^{\mathrm{kg}} \mathrm{kg}^{-1}$, and $\gamma_{A D}=1.8 \times 10^{10}[l]^{3}[\mathrm{~m}]^{-1}[t]^{-1}$, respectively. In this manner, the numerical values of $\epsilon$ and $\epsilon^{\prime}$ are $1.8 \times 10^{-9}[l]^{-3 / 2}[\mathrm{~m}]^{1 / 2}$ and $3.5 \times 10^{-17}[l]^{-15 / 2}[\mathrm{~m}]^{5 / 2}$, respectively. The magnetic field is scaled in units such that the constant $\mu_{0}$ is unity. Since the magnetic flux density has dimensions

$$
[B]=\frac{[m]}{[t][\text { charge }]}
$$


while $\mu_{0}$ has dimensions

$$
\left[\mu_{0}\right]=\frac{[\mathrm{m}][l]}{[\operatorname{charge}]^{2}},
$$

specifying $\mu_{0}=1$ therefore scales the magnetic field equal to $[B]=5.1 \mathrm{nT}$. With aforementioned units, the thermal energy per unit mass (6) is represented by $8.3 \times 10^{-3}(5 X / 4+3 Y / 8) T$, the heating rates due to cosmic rays and ambipolar diffusion are $\Gamma_{C R}=1.1 \times 10^{-3}[u] /[t]$ and

$$
\Gamma_{A D, \alpha}=\gamma_{A D} \rho_{i, \alpha} v_{d, \alpha}^{2},
$$

respectively, and the parameters for the gas cooling function are

$$
\begin{aligned}
& \log \left(\frac{\Lambda_{\alpha}}{[u] /[t]}\right)=-4.48- 0.87\left(\log \frac{\rho_{\alpha}}{2.24 \times 10^{-4}}\right) \\
&- 0.14\left(\log \frac{\rho_{\alpha}}{2.24 \times 10^{-4}}\right)^{2}, \\
& \beta_{\alpha}=3.07-0.11\left(\log \frac{\rho_{\alpha}}{2.24 \times 10^{-4}}\right) \\
&-0.13\left(\log \frac{\rho_{\alpha}}{2.24 \times 10^{-4}}\right)^{2} .
\end{aligned}
$$

\subsection{Initial setting}

The initial conditions for this simulation are a parallel magnetic field directed perpendicular to the $z$-axis so that the initial ratio of magnetic to gas pressure is everywhere a constant $\left(\alpha_{0}=1\right)$, and a density profile given by the equation (28). The magnetic field is assumed to be frozen in the fluid of charged particles and the central density is assumed to be $\rho_{0}=2.24 \times 10^{-4}[\rho]$. We choose a molecular cloud which has a mass fraction of molecular hydrogen and helium $X=0.75$ and $Y=0.25$, respectively, and has an initial uniform temperature of $T_{0}=50 \mathrm{~K}$. We assume that the cloud slab is spread from $z=-78[l]$ to $z=+78[l]$ (according to Fig. 1). The initial values of the cooling and heating functions are shown in Figure 2. As presented in this figure, the isobaric thermal instability criterion,

$$
\frac{\partial \Lambda}{\partial \rho}>\frac{\partial \Gamma}{\partial \rho},
$$

is satisfied in the outer parts of the cloud, thus, these regions are thermally unstable while the inner part is stable as outlined by Nejad-Asghar (2007).

Implementation of the boundary particles in the diffusion processes is an important problem in two-fluid SPH simulation. In ambipolar diffusion process, the ion particles are 
physically diffused through the neutral fluid, thus, the ions will be bared in the boundary regions of the cloud (i.e. without any neutral particles in their neighbors). Both the cloud and boundary regions contain ion and neutral particles. The complete system is represented by $N$ discrete but smoothed SPH particles (i.e. Lagrangian sample points) so that coagulation of particles and fragmentation of the cloud is truthfully revealed. Since it is desirable to have initially the same numerical resolution for both fluid components, we use $N / 2$ ions and $N / 2$ neutral particles. We set up boundary particles ( $4 h_{1}$ up and down in $z$ ) using the linear extrapolation approach (from the values of the inner particles) to attribute the appropriate drift velocity, drag acceleration, pressure acceleration, energy rate, and the magnetic induction rate to the boundary particles. We check the position of ions before making a tree and nearest neighbor search, so that we do not consider the bared ion particles in the boundary regions of the simulation at next time-step.

The present SPH code has the main features of the TreeSPH class so that the nearest neighbors searching are calculated by means of this procedure. The selection of time-step, $\Delta t$, is of great importance. There are several time-scales that can be defined locally in the system. For each particle 1, we calculate the smallest of these time-scales using its smallest smoothing length, $h_{1}$, i.e.

$$
\Delta t_{1}=C_{\text {cour }} \min \left[\frac{h_{1}}{\left|v_{1}\right|}, \frac{h_{1}}{v_{A, 1}}, \frac{h_{1}}{c_{s, 1}}\right]
$$

where $v_{A}=B / \sqrt{\mu_{0} \rho_{i}}$ is the Alfvén speed of ion fluid and $C_{\text {cour }}$ is the Courant number which in this paper is adopted equal to 0.3 (for numerical stability). The evolution were carried out to time $\langle u\rangle /\langle\Lambda, \Gamma\rangle \sim 0.43 / 0.025=17.2[t]$ so that the fragmentation of the cloud via thermal instability may be revealed.

\subsection{Results}

The molecular cloud has an initial uniform temperature of $T_{0}=50 \mathrm{~K}$. The Fig. 2 shows that the cooling is greater than heating at initial time $t=0$, but by passing the time, the drift speed of ions in the outer layers increases, thus, the heating rate growths in those regions. Increasing of the heating rate in the outer layers causes to stabilize those regions, and the intermediate layers become thermally unstable. The evolution of cooling and heating rates, causes to increase the temperature of the slab at outer regions while decrease the temperature at the intermediate parts of it. The temperature profiles at times $t=3.5[t], 10.5[t]$ and $17.2[t]$ are shown in Figure 3. This figure shows that the outer layers of the slab heat up, the intermediate layers cool down, and the inner layers hardly change. It is obvious that the instability of the cloud at the intermediate parts, causes the formation 
of two relative cool regions in those areas.

Since, in course of time, the thermal instability is shifted to the intermediate regions of the slab, the condensation mechanism is occurred there as depicted in Fig. 4. The growth of thermal instability results a density imbalance between the preformed condensations and their adjacent medium. As a result of this instability process, the particles are dragged from the adjoining of the condensations so that the density fluctuations growth and the fragmentation of the parent slab into cold, dense, low-mass cloudlets may be appeared. To compare the effect of the thermal instability, we firstly perform the isothermal simulation that have initially $T=50 \mathrm{~K}$. The thermal and density evolution of the slab are carried out to times $t=3.5[t], 10.5[t]$ and $17.2[t]$. The isothermal density profile and the density comparison with the isothermal case, are shown in Figure 4. This figure shows the resulting isothermal density evolution at the top panel while the density contrast, relative to the isothermal case, is shown in the bottom panel. In this run, the thermal instability is fully developed and makes density fluctuations in the intermediate parts of the cloud.

\section{Summary and conclusions}

Molecular clouds have a hierarchical structure that extends from the scale of the cloud down to much smaller masses for unbound structures. A weakly ionized self-gravitating one dimensional slab geometry is assumed in order to gain some insight into the fragmentation process. The two-fluid SPH technique was used to investigate the nonlinear thermal evolution of the slab. The initial conditions for this simulation are an inverse square cosine-hyperbolic profile for initial density and a parallel magnetic field directed perpendicular to the slab.

The initial values of the cooling and heating functions are shown in Figure 2. According to this figure, the isobaric thermal instability criterion is satisfied in the outer parts of the cloud. In course of time, the thermal instability is shifted to the intermediate regions of the slab so that the condensation mechanism is occurred there, thus, these regions are thermally unstable while the outer part is stable. The evolution were carried out to time $17.2[t]$ so that the fragmentation of the cloud via thermal instability is revealed.

The cooling and heating rates cause to increase the temperature of the clouds at outer regions while decrease the temperature at the inner part of it. It is obvious that the instability of the cloud at the intermediate parts, causes the formation of two relative cool regions in those areas, which is shown in Figure 3. The rapid growth of thermal instability results a density imbalance between the cloud and the surroundings. The isothermal density profile and density contrast, relative to the isothermal case, are shown in Figure 4. In this run, 
the thermal instability can fully develop and makes density fluctuations in the intermediate parts of the cloud.

This feature may be responsible for the planet formation in the intermediate parts of a collapsing molecular cloud and/or may also be liable for the formation of star forming dense cores in the clumps. The macro-velocity fields in the molecular clouds are highly turbulent and supersonic. It is then of uppermost importance to consider both collision and merger of the formed condensations. Merging is possibly the main onset mechanism to form dense cores, which likely evolve to star formation.

\section{Acknowledgments}

This work has been supported by Research Institute for Astronomy and Astrophysics of Maragha (RIAAM).

\section{REFERENCES}

André, P., Hennebelle, P., Peretto, N., 2008, Ap\&SS, 313, 29

Black, D.C., Scott, H., 1982, ApJ, 263, 696

Birk, G.T., 2000, Phys. Plasma, 7.3811

Boissé, P., Le Petit, F., Rollinde, E., Roueff, E., Pineau des Forêts, G., Andersson, B.G., Gry, C., Felenbok, P., 2005, A\&A, 429, 509

Churchwell, E., Whitney, B.A., Babler, B.L., Indebetouw, R., Meade, M.R., Watson, C., Wolff, M.J., Wolfire, M.G., Bania, T.M., Benjamin, R.A., and 12 coauthors, 2004, ApJS, 154, 322

Elmgreen, B.G., 2007, ApJ, 668, 1064

Falle, S.A.E.G., Ager, M., Hartquist, T.W., 2006, ASPC, 359, 137

Fiedler, R.A., Mouschovias, T.C., 1992, ApJ, 391, 199

Folini, D., Heyvaerts, J., Walder, R., 2004, A\&A, 414, 559

Fukue, T., Kamaya, H., 2007, ApJ, 669, 363

Gilden, D.L., 1984, ApJ, 283, 679 
Goldsmith, P.F., 2001, ApJ, 557, 736

Hosking, J.G., Whitworth, A.P., 2004, MNRAS, 347, 994 (HW)

Langer, W.D., Velusamy, T., Kuiper, T.B.H., Levin, S., Olsen, E., Migenes, V., 1995, ApJ, 453,293

Larson, R.B., 1985, MNRAS, 214, 379

MacLow, M.M., Norman, M.L., Konigl, A., Wardle, M., 1995, ApJ, 442, 726

Monaghan, J.J., 1992, ARA\&A, 30, 543

Nejad-Asghar, M., Ghanbari, J., 2003, MNRAS, 345, 1323

Nejad-Asghar, M., 2007, MNRAS, 379, 222

Nejad-Asghar, M., Khesali, A.R., Soltani, J., 2008, Ap\&SS, 313, 425

Pan, K., Federman, S.R., Welty, D.E., 2001, ApJ, 558, 105

Press, W.H., Teukolsky, S.A., Vetterling, W.T., Flannery, B.P., 1992, Nmerical Recipes, 2nd ed., Cambridge University

Shu, F., 1983, ApJ, 273, 202

Zuckerman, B., Palmer, P., 1974, ARA\&A, 12, 279 


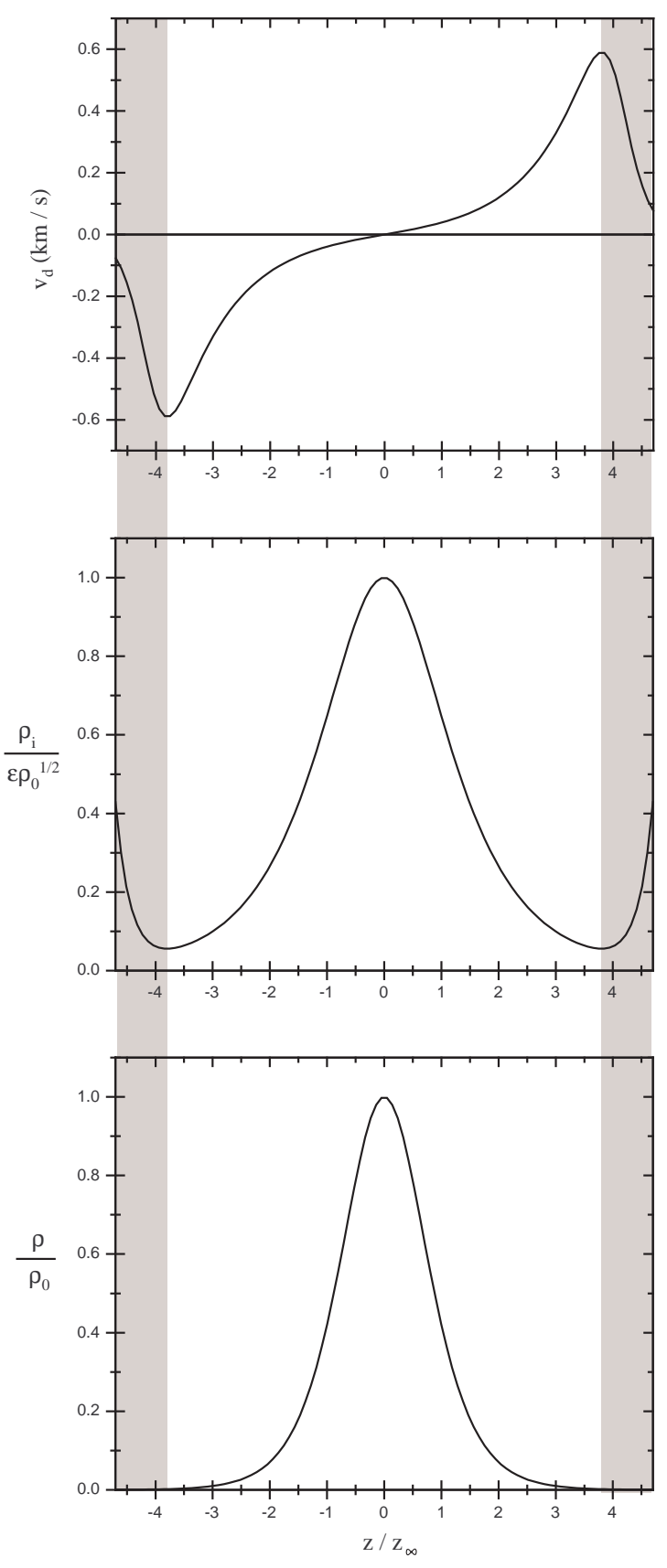

Fig. 1. - The initial drift velocity, ion density, and neutral density in the cloud and outercloud medium (gray region) for $\rho_{0}=3.8 \times 10^{-15} \mathrm{~kg} \cdot \mathrm{m}^{-3}, a=0.55 \mathrm{~km} \cdot \mathrm{s}^{-1}$, and $\alpha_{0}=1$. 


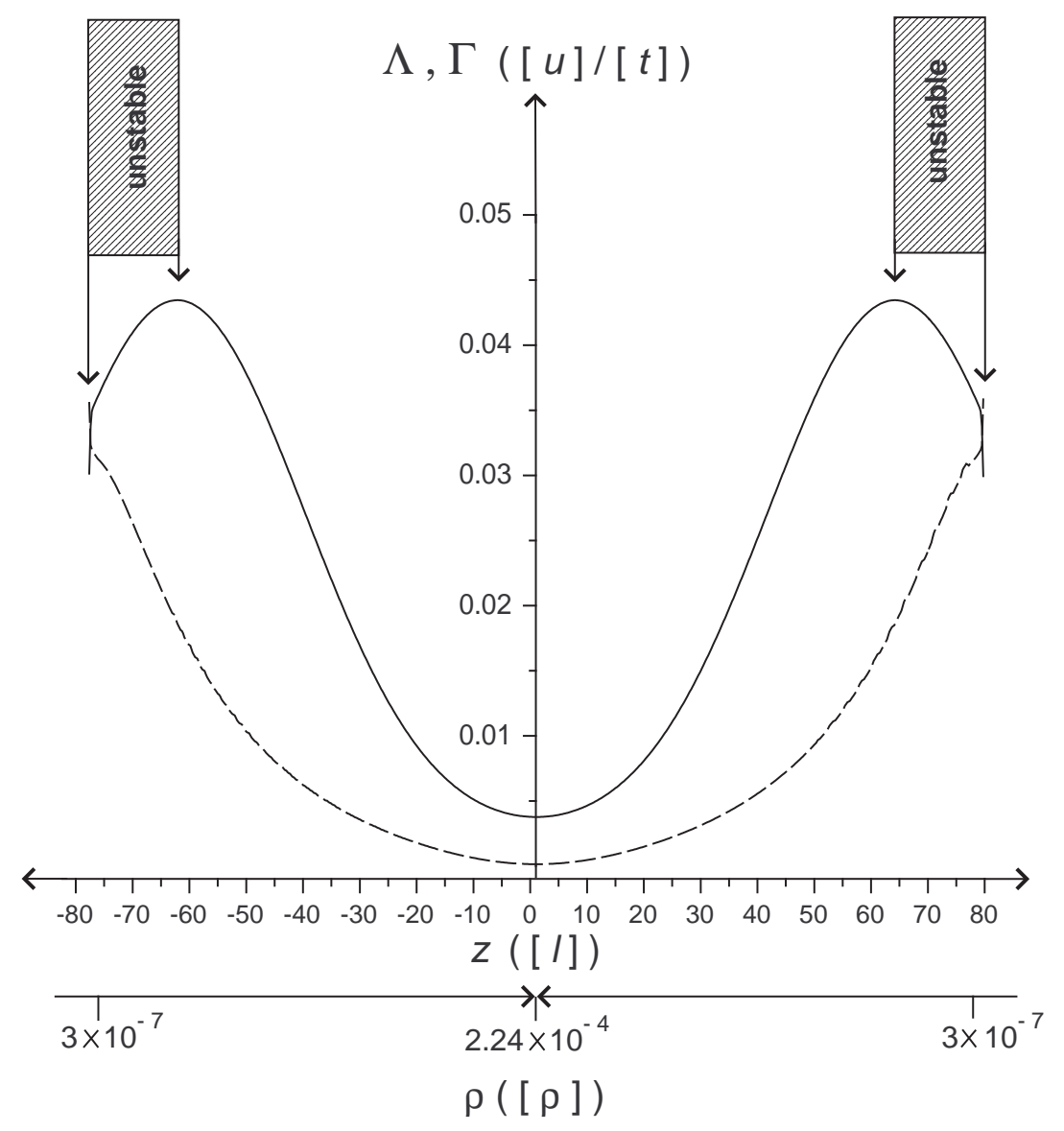

Fig. 2.- The initial values of the cooling (solid) and heating (dash) functions versus position and neutral density. 

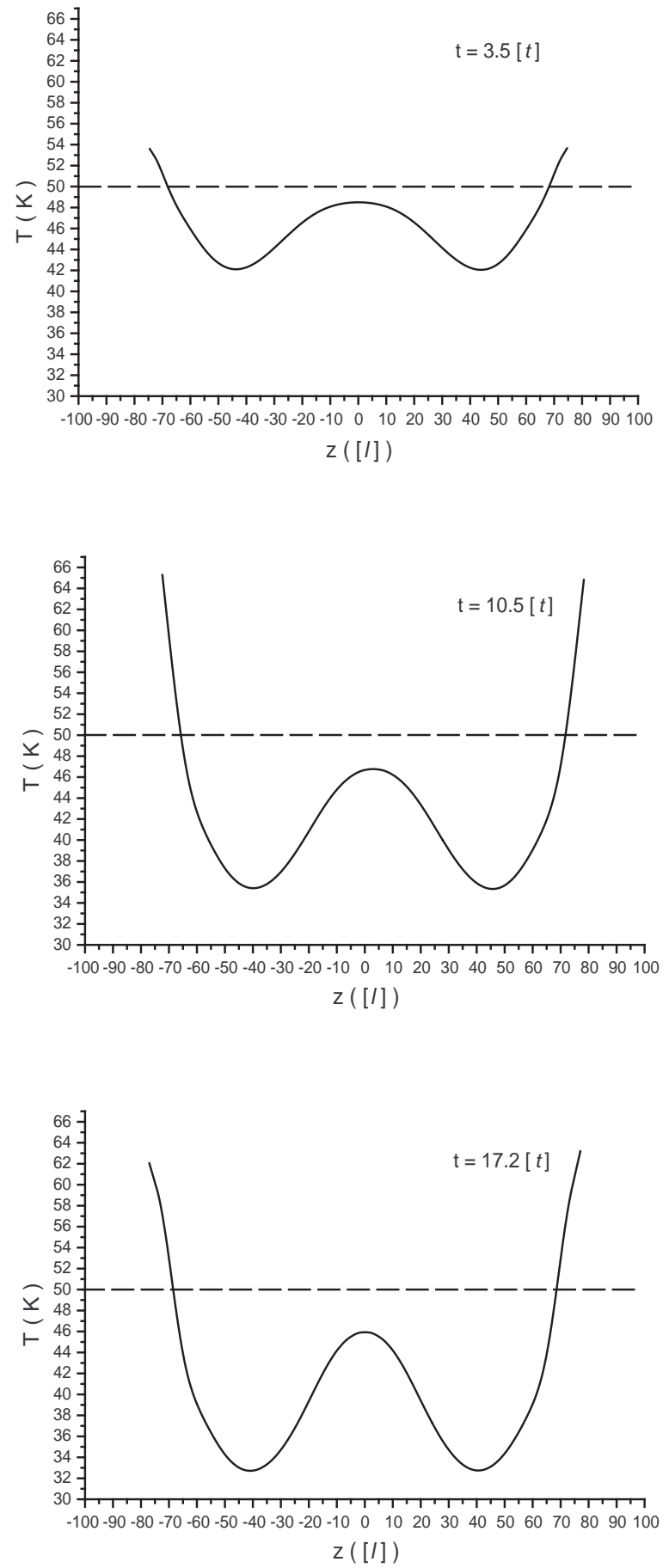

Fig. 3. - The temperature profile versus position at times $t=3.5[t], 10.5[t]$ and $17.2[t]$. The initial temperature of the slab is chosen as uniform which is shown by dash line. 

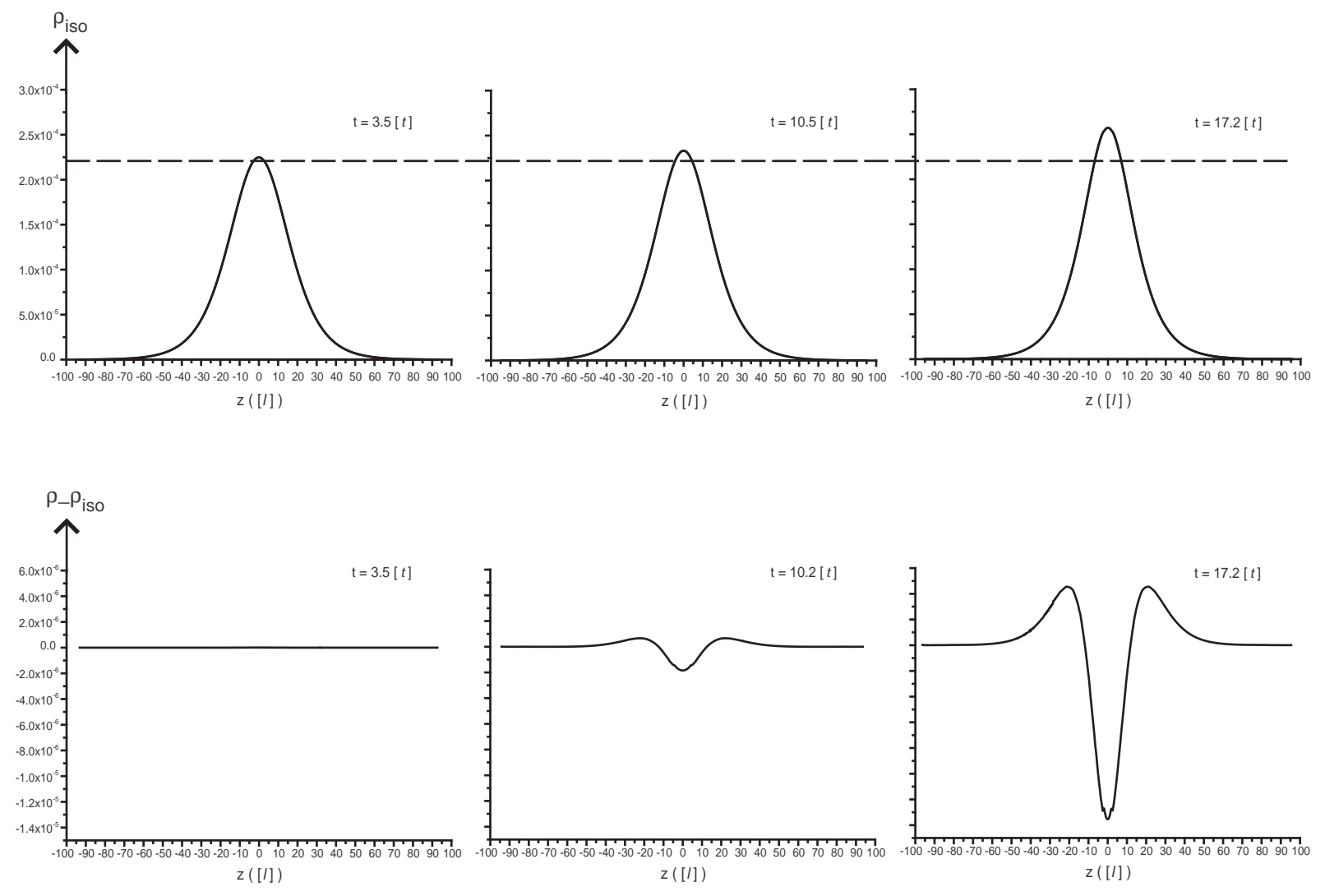

Fig. 4.- The isothermal density profile versus position at times $t=3.5[t], 10.5[t]$ and $17.2[t]$ (top panel), and the density comparison with the isothermal case at those times (bottom panel). The dash line in the top panel is the initial central density of the cloud. 